\title{
3.3. VARIATION SÉCULAIRE DES LONGITUDES
}

\author{
Nicolas Stoyko \\ (Observatoire de Paris, France)
}

\begin{abstract}
RÉSUMÉ
La réduction des résultats de services horaires dans un système uniforme permet de déterminer la variation séculaire des longitudes avec une grande précision. Il faut considérer un long intervalle de temps pour supprimer l'influence des variations quasi-périodiques dans la détermination des longitudes. On remarque des variations concommitantes des longitudes des stations rapprochées, se trouvant sur un même bloc de l'écorce terrestre, ou des variations de sens opposé, quand les stations étudiées se trouvent sur des blocs voisins, se déplaçant l'un par rapport à l'autre.
\end{abstract}

\section{PEЗЮME}

Приведение результатов служб времени к одной системе позволяет определить вековое изменение долгот с большой точностью. Необходимо использовать большой промежуток времени, чтобы уничтожить влияние квази-периодических вариаций в определении долгот. Замечено одновременное изменение долгот близлежащих станций, находящихся на одном блоке земной коры, а также движения в противоположных направлениях станций, находяшихся на соседних блоках, которые перемещаются один по отношению к другому.

\section{ABSTRACT}

The reduction of time service results on a uniform system permits the secular determination of longitudes with high precision. A long interval of time is needed to eliminate quasi-periodic effects. One notes that the variations are similar when stations are in the same terrestrial region and opposed when in different regions.

Nous avons révisé les résultats de services horaires de 1925 à 1965 en prenant en considération les nouvelles valeurs de longitudes conventionnelles et le catalogue FK4. Nous avons ramené ces résultats à l'Observatoire moyen composé de 11 services horaires ayant travaillé pendant toute la période étudiée. Sur les Figures 1 et 2 nous avons représenté les courbes des corrections des longitudes ainsi calculées pour chaque observatoire. Nous avons déterminé d'après ces valeurs les corrections de longitudes pour l'année $1965\left(\Delta \lambda_{65}\right)$ et leurs variations annuelles $\left(\Delta m_{a}\right)$ qui figurent dans le Tableau 1. En plus ce tableau contient la variation annuelle des longitudes déterminée d'après la méthode graphique $\left(\Delta m_{a g}\right)$.

Comme les variations de longitudes ne sont pas tout-à-fait linéaires, nous publions dans le Tableau 2 les marches annuelles de longitudes de 10 en 10 ans et leurs écarts moyens $(\mathrm{Em})$. La dernière colonne $\left(\mathrm{M}_{40}\right)$ contient la moyenne de 40 années. On voit que les variations annuelles de longitudes pour les différentes périodes sont très 


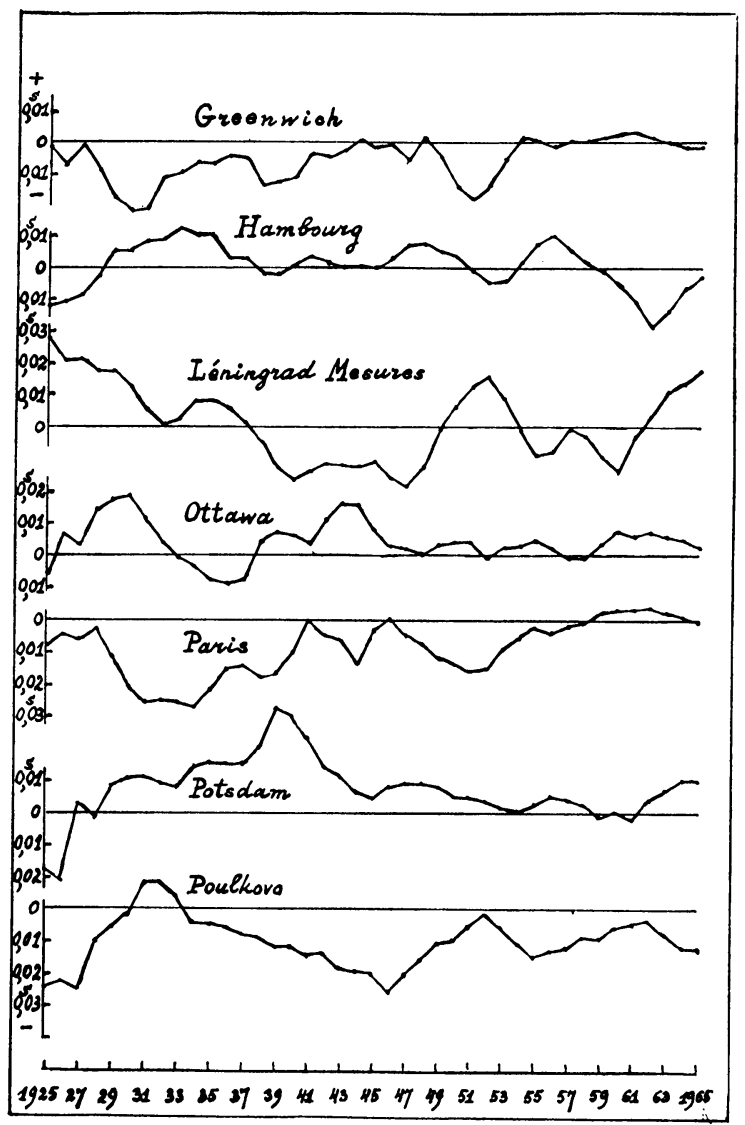

FIG. 1. Variations séculaires des longitudes.

discordantes (de l'ordre de 0 ".0006), ce qui explique les conclusions quelquefois contradictoires.

La variation annuelle de longitudes entre l'Amérique du Nord et l'Europe pour la période de 1925 à 1965 est de -0 ".00060 (rapprochement des continents). Pour le contrôle nous avons traité les résultats de la détermination des longitudes à partir de 1871, réduits au même système et n'entrant pas dans le service courant de l'heure (Tableau 3). Ces résultats, couvrant la période de 88 ans, donnent la marche annuelle de $-0 " .00068$, ce qui est en bon accord avec la valeur précédente.

Selon Wegener il devrait exister un déplacement important de Madagascar par rapport à l'Afrique du Sud (éloignement) et celui de Groenland vers l'Ouest. D'après Stoyko (1966) la variation annuelle de la longitude de Madagascar vers l'Est est de -0.00033 .

D'après les deux premières opérations des longitudes mondiales le déplacement 


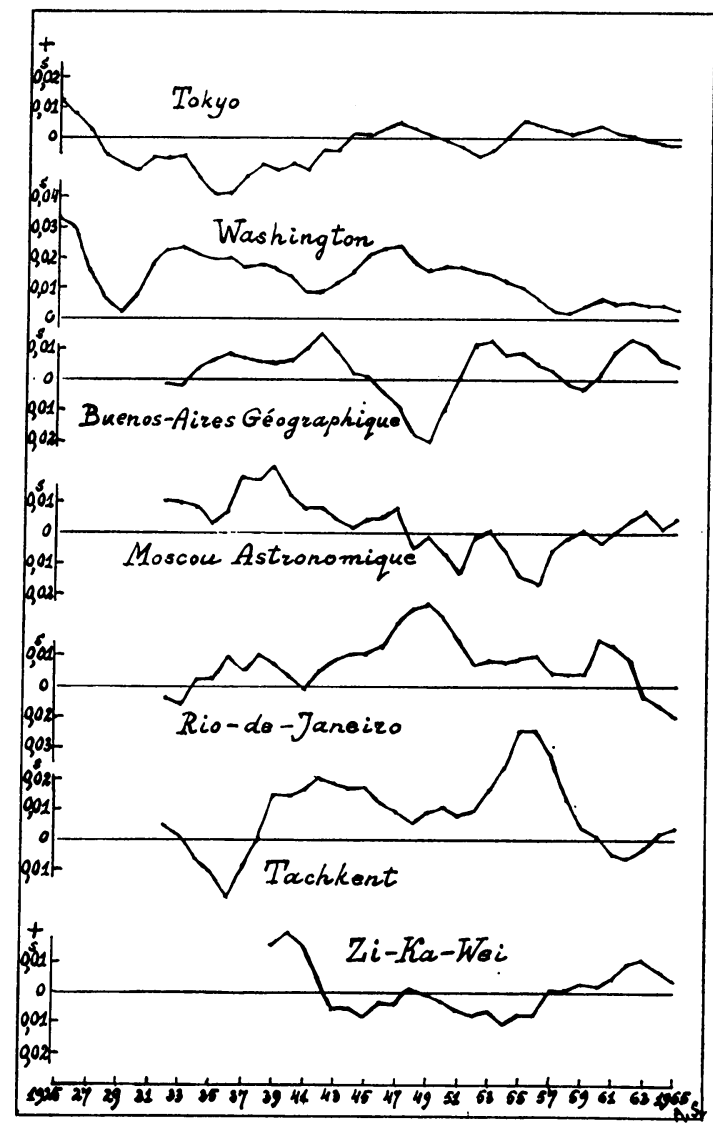

FIG. 2. Variations séculaires des longitudes.

annuel du Cap vers l'Est est égal à - 0 ".00148, ce qui correspond à la diminution de la distance entre l'Afrique du Sud et le Madagascar (43 cm par an).

La détermination moderne de la longitude de la station de Kornok (Groenland) a été faite en 1927 (Sindig, 1955). Les observations de contrôle, faites en 1948, ont donné une valeur supérieure de 0".02 par rapport à celle de 1927, ce qui indique un déplacement faible de Groenland vers l'Ouest (19 cm par an).

Les mouvements récents de l'écorce terrestre se produisent par blocs qui peuvent avoir d'assez vastes dimensions. Dans ce cas les stations qui se trouvent sur un même bloc doivent avoir les variations concommitantes de longitudes. Nous avons étudié dans ce but le mouvement des couples suivants de stations: Greenwich-Paris, Hambourg-Potsdam, Léningrad-Poulkovo et Ottawa-Washington.

En éliminant les variations séculaires de longitudes de Greenwich et de Paris d'après le Tableau 1, nous avons trouvé que les variations résiduelles de longitudes à courte 


\section{Tableau 1}

Corrections des longitudes pour l'année 1965 et leurs variations annuelles par rapport à l'Observatoire moyen constant

(En millièmes de seconde de temps)

\begin{tabular}{|c|c|c|c|c|c|c|c|}
\hline \multirow[b]{2}{*}{ Noms } & \multirow[b]{2}{*}{$\mathrm{Abr}^{\mathrm{n}}$} & \multirow[b]{2}{*}{$n$} & \multicolumn{4}{|c|}{ Moindres carrés } & \multirow{2}{*}{$\begin{array}{c}\text { Graph } \\
\Delta m_{a g}\end{array}$} \\
\hline & & & $\Delta \lambda_{65}$ & $\mathrm{Em}$ & $\Delta m_{a}$ & $\mathrm{E} m_{a}$ & \\
\hline Buenos Aires g. & $\mathrm{BAg}$ & 34 & +4.4 & $\pm 3 \cdot 7$ & +0.05 & \pm 0.14 & $+0 \cdot 10$ \\
\hline Greenwich & Gr & 41 & +0.5 & $1 \cdot 7$ & +0.30 & 7 & +0.25 \\
\hline Hambourg & $\mathrm{H}$ & 41 & -2.4 & $2 \cdot 2$ & -0.16 & 9 & -0.25 \\
\hline Léningrad mes. & $\mathrm{Lm}$ & 41 & -4.5 & $3 \cdot 7$ & -0.29 & 16 & -0.32 \\
\hline Moscou astr. & $\mathrm{Ma}$ & 34 & $-5 \cdot 1$ & $3 \cdot 3$ & -0.49 & 13 & -0.38 \\
\hline Neuchâtel & $\mathrm{N}$ & 41 & $-2 \cdot 1$ & $5 \cdot 2$ & -0.97 & 22 & $"$ \\
\hline Ottawa & $\mathbf{O}$ & 41 & $+4 \cdot 1$ & 1.9 & -0.03 & 8 & -0.27 \\
\hline Paris & $\mathrm{Pa}$ & 41 & +1.0 & $2 \cdot 1$ & +0.46 & 9 & +0.40 \\
\hline Potsdam & $\mathrm{Pt}$ & 41 & +5.9 & $2 \cdot 7$ & -0.09 & 12 & -0.05 \\
\hline Poulkovo & $\mathrm{Pu}$ & 41 & -9.8 & $2 \cdot 4$ & +0.01 & 11 & -0.05 \\
\hline Rio de Janeiro & $\mathbf{R J}$ & 34 & $+8 \cdot 4$ & $3 \cdot 8$ & +0.03 & 15 & $+0 \cdot 10$ \\
\hline Tashkent & $\mathrm{Ta}$ & 34 & $+12 \cdot 5$ & $5 \cdot 5$ & +0.23 & 22 & +0.37 \\
\hline Tokyo & To & 41 & +1.9 & $2 \cdot 0$ & +0.22 & 9 & +0.12 \\
\hline Uccle & $\mathbf{U}$ & 41 & -5.4 & $7 \cdot 9$ & +0.88 & 34 & $"$ \\
\hline Washington & W & 41 & $+6 \cdot 2$ & 1.9 & -0.40 & 8 & -0.52 \\
\hline Zi-Ka-Wei & $\mathbf{Z i}$ & 27 & +0.2 & $5 \cdot 7$ & -0.06 & 20 & -0.20 \\
\hline
\end{tabular}

Tableau 2

Variations annuelles des longitudes de 10 en 10 ans et leur moyenne (En millièmes de seconde de temps)

Marche annuelle

\begin{tabular}{|c|c|c|c|c|c|c|}
\hline $\mathrm{Abr}^{\mathrm{n}}$ & $\begin{array}{l}1926 \\
1935\end{array}$ & $\begin{array}{l}1936 \\
1945\end{array}$ & $\begin{array}{l}1946 \\
1955\end{array}$ & $\begin{array}{l}1956 \\
1965\end{array}$ & $\mathrm{Em}$ & $M_{40}$ \\
\hline BAg & " & -0.31 & +2.97 & +0.79 & \pm 0.54 & $+1 \cdot 15$ \\
\hline Gr & $-0 \cdot 10$ & +0.88 & 0.00 & -0.09 & $\cdot 18$ & +0.17 \\
\hline $\mathbf{H}$ & $+2 \cdot 51$ & -0.16 & -0.55 & -2.04 & .72 & -0.06 \\
\hline Lm & -2.03 & -1.69 & $+2 \cdot 11$ & +2.67 & .76 & +0.27 \\
\hline Ma & " & -1.50 & -1.56 & +1.80 & .44 & -0.42 \\
\hline $\mathrm{N}$ & +3.29 & -3.09 & -2.01 & -0.69 & .78 & -0.63 \\
\hline O & -1.78 & +2.29 & +0.13 & +0.51 & .29 & +0.29 \\
\hline $\mathbf{P a}$ & -2.76 & +1.50 & -0.32 & +0.42 & .24 & -0.29 \\
\hline $\mathrm{Pt}$ & +2.77 & -1.57 & -1.00 & +0.76 & .42 & +0.24 \\
\hline $\mathrm{Pu}$ & +2.59 & -1.66 & $+1 \cdot 56$ & +0.20 & .39 & +0.67 \\
\hline RJ & " & +0.13 & -1.65 & -1.70 & .78 & -0.74 \\
\hline $\mathrm{Ta}$ & " & +3.67 & +2.22 & $-3 \cdot 52$ & 1.05 & +0.79 \\
\hline To & -2.09 & +1.97 & -0.43 & -0.70 & $\cdot 13$ & -0.31 \\
\hline $\mathbf{U}$ & -2.91 & +0.81 & +1.49 & -2.67 & .35 & -0.82 \\
\hline W & $+1 \cdot 17$ & -0.33 & -1.29 & -0.03 & $\cdot 16$ & -0.12 \\
\hline $\mathrm{Zi}$ & & -5.04 & -0.85 & +1.35 & \pm 0.37 & -1.51 \\
\hline
\end{tabular}


Tableau 3

Corrections des différences de longitudes entre l'Amérique du Nord et l'Europe occidentale réduites à un même système et aux nouvelles longitudes conventionnelles

\begin{tabular}{|c|c|c|c|}
\hline & W-Eu & & W-Eu \\
\hline 1871 & $+0 " .0630$ & 1926 & +0.0095 \\
\hline 1892 & $+\quad 450$ & 1933 & $+\quad 193$ \\
\hline 1914 & 180 & 1959 & 030 \\
\hline 1922 & 179 & & \\
\hline
\end{tabular}

\section{Tableau 4}

Variations annuelles des longitudes entre les continents d'après les longitudes mondiales et les services horaires permanents

\begin{tabular}{|c|c|c|}
\hline & Long. mond. & Service perm. \\
\hline Période & $1930,3-1958,8$ & $1925-1965$ \\
\hline W-Eu4 & $-0 " .00030$ & -0.00055 \\
\hline Eu4-To & $-\quad 28$ & $-\quad 07$ \\
\hline To-W & 58 & 62 \\
\hline
\end{tabular}

durée sont, en majorité de cas, du même sens ( $75 \%$ ). Le coefficient de corrélation entre leurs mouvements est $r=+0 \cdot 6$. Comme ces deux observatoires sont complétement indépendants l'un de l'autre, on peut supposer qu'ils se trouvent sur un même bloc de l'écorce terrestre. La même conclusion est valable pour les autres couples de l'Europe.

Dans le cas des observatoires d'Ottawa et de Washington on remarque qu'après l'élimination du terme séculaire, leurs mouvements à courte période sont de sens opposé. Le coefficient de corrélation est $r=-0 \cdot 6$. Cela peut provenir du fait que ces observatoires se trouvent sur les deux blocs différents de l'écorce terrestre, dont la ligne de séparation est dirigée dans le sens WS-EN (Grands lacs, fleuve St. Laurent). Si les longitudes du bloc Sud diminuent (mouvement vers l'Est), les latitudes doivent augmenter en raison du glissement de deux blocs le long de la ligne de la séparation. Les résultats de la station de latitude de Gaithersburg, indépendants du catalogue d'étoiles, montrent d'après l'étude de Stoyko (1967) l'existence du déplacement de cette station vers le Nord de 0".003 par an, ce qui est en accord avec la variation de longitude de Washington vers l'Est. Nous avons trouvé un coefficient de corrélation très fort $r=-0.92$ entre la variation de longitude de Washington et de latitude de Gaithersburg.

Dans le Tableau 4 nous donnons les conclusions sur le mouvement séculaire des continents d'après les résultats des trois opérations des longitudes mondiales (Stoyko, 1966). Les résultats obtenus montrent l'existence d'un rapprochement entre l'Amérique du Nord et l'Europe, ainsi qu'entre l'Europe et le Japon et d'un éloignement entre 
le Japon et l'Amérique du Nord (élargissement de l'Océan Pacifique). Dans la partie droite du Tableau 4 nous donnons les résultats obtenus d'après les services horaires permanents, confirmant la conclusion précedente.

\section{Références}

Sindig, E. (1955) Mém. Inst. Géodés. Danemark, 19, 19.

Stoyko, Mme A. (1966) Ann. Intern. Geophys. Year, 42, 1-369.

Stoyko, Mme A. (1968) the present volume, p. 52. 